\title{
SPOT DIAGNOSIS ANSWER
}

\section{Answer}

Diagnosis: pulmonary aspergilloma of the right apex

Name of sign: Monod Sign

This sign is sometimes called: air crescent sign, although strictly speaking, air crescent sign is found in invasive pulmonary aspergillosis which is recovering.

The mass in the cavity will change position if the HRCT is repeated in prone position (thereby proving that it is a mobile mass). 